\title{
Vertex-oriented Hamilton cycles in directed graphs
}

\author{
Michael J. Plantholt \\ Department of Mathematics \\ Illinois State University \\ Normal, IL 61790-4520, USA \\ mikep@ilstu.edu
}

\author{
Shailesh K. Tipnis \\ Department of Mathematics \\ Illinois State University \\ Normal, IL 61790-4520 \\ tipnis@ilstu.edu
}

Submitted: Feb 16, 2009; Accepted: Sep 12, 2009; Published: Sep 18, 2009

Mathematics Subject Classifications: 05C20, 05C45

\begin{abstract}
Let $D$ be a directed graph of order $n$. An anti-directed Hamilton cycle $H$ in $D$ is a Hamilton cycle in the graph underlying $D$ such that no pair of consecutive arcs in $H$ form a directed path in $D$. We prove that if $D$ is a directed graph with even order $n$ and if the indegree and the outdegree of each vertex of $D$ is at least $\frac{2}{3} n$ then $D$ contains an anti-directed Hamilton cycle. This improves a bound of Grant [7]. Let $V(D)=P \cup Q$ be a partition of $V(D)$. A $(P, Q)$ vertex-oriented Hamilton cycle in $D$ is a Hamilton cycle $H$ in the graph underlying $D$ such that for each $v \in P$, consecutive arcs of $H$ incident on $v$ do not form a directed path in $D$, and, for each $v \in Q$, consecutive $\operatorname{arcs}$ of $H$ incident on $v$ form a directed path in $D$. We give sufficient conditions for the existence of a $(P, Q)$ vertex-oriented Hamilton cycle in $D$ for the cases when $|P| \geqslant \frac{2}{3} n$ and when $\frac{1}{3} n \leqslant|P| \leqslant \frac{2}{3} n$. This sharpens a bound given by Badheka et al. in [1].
\end{abstract}

\section{Introduction}

Let $G$ be a graph with vertex set $V(G)$ and edge set $E(G)$. For a vertex $v \in V(G)$, the degree of $v$ in $G$, denoted by $\operatorname{deg}(v, G)$ is the number of edges of $G$ incident on $v$. Let $\delta(G)=\min _{v \in V(G)}\{\operatorname{deg}(v, G)\}$. Let $D$ be a directed graph with vertex set $V(D)$ and arc set $A(D)$. For a vertex $v \in V(D)$, the outdegree (respectively, indegree) of $v$ in $D$ denoted by $d^{+}(v, D)$ (respectively, $d^{-}(v, D)$ ) is the number of arcs of $D$ directed out of $v$ (respectively, directed into $v)$. Let $\delta^{0}(D)=\min _{v \in V(D)}\left\{\min \left\{d^{+}(v, D), d^{-}(v, D)\right\}\right\}$. The graph underlying $D$ is the graph obtained from $D$ by ignoring the directions of the arcs of $D$. A directed Hamilton cycle $H$ in $D$ is a Hamilton cycle in the graph underlying $D$ such that all pairs of consecutive $\operatorname{arcs}$ in $H$ form a directed path in $D$. An anti-directed Hamilton cycle $H$ in $D$ is a Hamilton cycle in the graph underlying $D$ such that no pair of consecutive $\operatorname{arcs}$ in $H$ 
form a directed path in $D$. Note that if $D$ contains an anti-directed Hamilton cycle then $|V(D)|$ must be even. Let $D$ be a directed graph, and let $V(D)=P \cup Q$ be a partition of $V(D)$. A $(P, Q)$ vertex-oriented Hamilton cycle in $D$ is a Hamilton cycle $H$ in the graph underlying $D$ such that for each $v \in P$, consecutive arcs of $H$ incident on $v$ do not form a directed path in $D$, and, for each $v \in Q$, consecutive arcs of $H$ incident on $v$ form a directed path in $D$. Note that if $D$ contains a $(P, Q)$ vertex-oriented Hamilton cycle then $|P|$ must be even. The idea of a $(P, Q)$ vertex-oriented Hamilton cycle generalizes the ideas of a directed Hamilton cycle and an an anti-directed Hamilton cycle, because a directed Hamilton cycle in $D$ is a $(\emptyset, V(D))$ vertex-oriented Hamilton cycle in $D$ and an anti-directed Hamilton cycle in $D$ is a $(V(D), \emptyset)$ vertex-oriented Hamilton cycle in $D$. We refer the reader to $([1,2,5])$ for all terminology and notation that is not defined in this paper.

The following classical theorems by Dirac [3] and Ghouila-Houri [6] give sufficient conditions for the existence of a Hamilton cycle in a graph $G$ and for the existence of a directed Hamilton cycle in a directed graph $D$ respectively.

Theorem 1 [3] If $G$ is a graph of order $n \geqslant 3$ and $\delta(G) \geqslant \frac{n}{2}$, then $G$ contains a Hamilton cycle.

Theorem 2 [6] If $D$ is a directed graph of order $n$ and $\delta^{0}(D) \geqslant \frac{n}{2}$, then $D$ contains a directed Hamilton cycle.

The following theorem by Grant [7] gives a sufficient condition for the existence of an anti-directed Hamilton cycle in a directed graph $D$.

Theorem 3 [7] If $D$ is a directed graph with even order $n$ and if $\delta^{0}(D) \geqslant \frac{2}{3} n+\sqrt{n \log (n)}$ then $D$ contains an anti-directed Hamilton cycle.

In his paper Grant [7] conjectured that the theorem above can be strengthened to assert that if $D$ is a directed graph with even order $n$ and if $\delta^{0}(D) \geqslant \frac{1}{2} n$ then $D$ contains an anti-directed Hamilton cycle. Mao-cheng Cai [10] gave a counter-example to this conjecture. However, the following theorem by Häggkvist and Thomason [8] proves that Grant's conjecture is asymptotically true.

Theorem 4 [8] There exists an integer $N$ such that if $D$ is a directed graph of order $n \geqslant N$ and $\delta^{0}(D) \geqslant\left(\frac{1}{2}+n^{-\frac{1}{6}}\right) n$ then $D$ contains an $n$-cycle with arbitrary orientation.

We point out here that if $D$ is an oriented graph (i.e. a digraph for which at most one of the $\operatorname{arcs}(u, v)$ and $(v, u)$ can be in $A(D))$ Häggkvist and Thomason [9] have obtained the following result.

Theorem 5 [9] For every $\epsilon>0$, there exists $N(\epsilon)$ such that if $D$ is an oriented graph of order $n \geqslant N(\epsilon)$ and $\delta^{0}(D) \geqslant\left(\frac{5}{12}+\epsilon\right) n$ then $D$ contains an $n$-cycle with arbitrary orientation.

In Section 2 of this paper we prove the following improvement of Theorem 3 by Grant [7]. 
Theorem 6 If $D$ is a directed graph with even order $n$ and if $\delta^{0}(D) \geqslant \frac{2}{3} n$ then $D$ contains an anti-directed Hamilton cycle.

In Section 3 of this paper we turn our attention to $(P, Q)$ vertex-oriented Hamilton cycles. In [1] the following theorem giving a sufficient condition for the existence of a $(P, Q)$ vertex-oriented Hamilton cycle was proved. For the sake of completeness we include the proof of this theorem in Section 3.

Theorem 7 [1] Let $D$ be a directed graph of order $n$ and let $V(D)=P \cup Q$ be a partition of $V(D)$. If $|P|=2 j$ for some integer $j \geqslant 0$, and $\delta^{0}(D) \geqslant \frac{n}{2}+j$, then $D$ contains a $(P, Q)$ vertex-oriented Hamilton cycle.

Let $D$ be a directed graph and let $D^{\prime}$ be the spanning directed subgraph of $D$ consisting of all arcs $u v \in A(D)$ for which $v u \in A(D)$. Let $G^{\prime}$ be the graph underlying $D^{\prime}$. We note that if $\delta^{0}(D) \geqslant \frac{3}{4} n$, then $\delta\left(G^{\prime}\right) \geqslant \frac{n}{2}$, and hence Theorem 1 implies that $G^{\prime}$ contains a Hamilton cycle. Thus, if $\delta^{0}(D) \geqslant \frac{3}{4} n$ and $|P|$ is even, then $D$ trivially contains a $(P, Q)$ vertex-oriented Hamilton cycle for any partition $V(D)=P \cup Q$ of $V(D)$.

In Section 3 of this paper we prove the following two theorems that give sufficient conditions for the existence of a $(P, Q)$ vertex-oriented Hamilton cycle that are sharper than the one given in Theorem 7 for the cases when $|P| \geqslant \frac{2}{3} n$ and when $\frac{1}{3} n \leqslant|P| \leqslant \frac{2}{3} n$.

Theorem 8 Let $D$ be a directed graph of order $n \geqslant 4$ and let $V(D)=P \cup Q$ be a partition of $V(D)$. If $|P|=2 j \geqslant \frac{2}{3} n$ for some integer $j \geqslant 0$, and $\delta^{0}(D) \geqslant \frac{n}{2}+\frac{j}{2}$, then $D$ contains $a(P, Q)$ vertex-oriented Hamilton cycle.

Theorem 9 Let $D$ be a directed graph of order $n \geqslant 4$ and let $V(D)=P \cup Q$ be a partition of $V(D)$. If $|P|=2 j$ for some integer $j \geqslant 0$ with $\frac{1}{3} n \leqslant 2 j \leqslant \frac{2}{3} n$ and $\delta^{0}(D) \geqslant \frac{2}{3} n$, then $D$ contains a $(P, Q)$ vertex-oriented Hamilton cycle.

\section{Proof of Theorem 6}

A partition of a set $S$ with $|S|$ being even into $S=X \cup Y$ is an equipartition of $S$ if $|X|=|Y|=\frac{|S|}{2}$. We will use the following theorem by Moon and Moser [11].

Theorem 10 [11] Let $G$ be a bipartite graph of even order $n$, with equipartition $V(G)=$ $X \cup Y$. If $x \in X, y \in Y, x y \notin E(G)$, and, $\operatorname{deg}(x)+\operatorname{deg}(y)>\frac{n}{2}$, then $G$ contains $a$ Hamilton cycle if and only if $G+x y$ contains a Hamilton cycle.

For a bipartite graph $G$ of order $n$, with partition $V(G)=X \cup Y$, the closure of $G$ is defined as the supergraph of $G$ obtained by iteratively adding edges between pairs of nonadjacent vertices $x \in X$ and $y \in Y$ whose degree sum is greater than $\frac{n}{2}$.

For an equipartition of $V(D)$ into $V(D)=X \cup Y$, let $B(X \rightarrow Y)$ be the bipartite directed graph with vertex set $V(D)$, equipartition $V(D)=X \cup Y$, and with $(x, y) \in A(B(X \rightarrow$ $Y)$ ) if and only if $x \in X, y \in Y$, and, $(x, y) \in A(D)$. Let $B(X, Y)$ denote the bipartite graph underlying $B(X \rightarrow Y)$. It is clear that $B(X, Y)$ contains a Hamilton cycle if and only if $B(X \rightarrow Y)$ contains an anti-directed Hamilton cycle. The following lemma will imply Theorem 6 . 
Lemma 1 If $D$ is a directed graph with even order $n$ and if $\delta^{0}(D) \geqslant \frac{2}{3} n$ then there exists an equipartition of $V(D)$ into $V(D)=X \cup Y$, such that $\mid\{v \in V(D): \operatorname{deg}(v, B(X, Y)) \geqslant$ $\left.\frac{1}{3} n\right\} \mid>\frac{n}{2}$.

Proof. For a vertex $v \in V(D)$, let $n_{1}(v)$ be the number of equipartitions of $V(D)$ into $V(D)=X \cup Y$ for which $\operatorname{deg}(v, B(X, Y)) \geqslant \frac{1}{3} n$ and let $n_{2}(v)$ be the number of equipartitions of $V(D)$ for which $\operatorname{deg}(v, B(X, Y))<\frac{1}{3} n$. We will show that $n_{1}(v)>n_{2}(v)$ for each $v \in V(D)$ which in turn clearly implies the conclusion in the lemma.

Since $n$ is even, we have that $n \equiv 0 \bmod 6$ or $n \equiv 2 \bmod 6$ or $n \equiv 4 \bmod 6$. We give the proof for the case in which $n \equiv 2 \bmod 6$; the other cases can be proved similarly.

Hence, assume that $|V(D)|=n=6 k+2$ for some positive integer $k$. Let $v$ be a vertex in $V(D)$. Now, $\delta^{0}(D) \geqslant \frac{2}{3} n$ implies that $d^{+}(v, D) \geqslant 4 k+2$, and since we wish to argue that $n_{1}(v)>n_{2}(v)$, we can assume that $d^{+}(v, D)=4 k+2$. Note that this implies that $\operatorname{deg}(v, B(X, Y)) \geqslant k+2$ for every equipartition of $V(D)$ into $V(D)=X \cup Y$. Now, $n_{1}(v)$ is the number of equipartitions of $V(D)$ into $V(D)=X \cup Y$ for which $2 k+2 \leqslant \operatorname{deg}(v, B(X, Y)) \leqslant 3 k+1$, and, $n_{2}(v)$ is the number of equipartitions of $V(D)$ into $V(D)=X \cup Y$ for which $k+2 \leqslant \operatorname{deg}(v, B(X, Y))<2 k+1$. Hence, because $v$ may be in $X$ or $Y$, we have that

$$
n_{1}(v)=2 \sum_{i=1}^{k}\left(\begin{array}{c}
4 k+2 \\
2 k+i+1
\end{array}\right)\left(\begin{array}{c}
2 k-1 \\
k-i
\end{array}\right)
$$

and that,

$$
n_{2}(v)=2 \sum_{i=1}^{k}\left(\begin{array}{c}
4 k+2 \\
2 k+2-i
\end{array}\right)\left(\begin{array}{c}
2 k-1 \\
k+i-1
\end{array}\right) .
$$

Since $\left(\begin{array}{c}4 k+2 \\ 2 k+i+1\end{array}\right)\left(\begin{array}{c}2 k-1 \\ k-i\end{array}\right)>\left(\begin{array}{c}4 k+2 \\ 2 k+2-i\end{array}\right)\left(\begin{array}{c}2 k-1 \\ k+i-1\end{array}\right)$ for each $i=1,2, \ldots, k$, we have that $n_{1}(v)>n_{2}(v)$ and this completes the proof of the lemma.

Proof of Theorem 6. As given by Lemma 1, consider an equipartition of $V(D)$ into $V(D)=X \cup Y$ such that $\left|\left\{v \in V(D): \operatorname{deg}(v, B(X, Y)) \geqslant \frac{1}{3} n\right\}\right|>\frac{n}{2}$. Let $Z=\left\{v \in V(D): \operatorname{deg}(v, B(X, Y)) \geqslant \frac{1}{3} n\right\}$ and let $X^{*}=X \cap Z$ with $\left|X^{*}\right|=k>0$, and let $Y^{*}=Y \cap Z$ with $\left|Y^{*}\right| \geqslant \frac{n}{2}-k+1$. Let $B^{+}(X, Y)$ denote the closure of $B(X, Y)$. Note that since $\delta^{0}(D) \geqslant \frac{2}{3} n$, we have that $\operatorname{deg}(v, B(X, Y))>\frac{n}{6}$ for each vertex $v$. Hence, $\operatorname{deg}\left(v, B^{+}(X, Y)\right)=\frac{n}{2}$ for each $v \in X^{*} \cup Y^{*}$. Therefore, $\operatorname{deg}\left(v, B^{+}(X, Y)\right) \geqslant \frac{n}{2}-k+1$ for each $v \in X$ and $\operatorname{deg}\left(v, B^{+}(X, Y)\right) \geqslant k$ for each $v \in Y$. Now, Theorem 10 implies that $B^{+}(X, Y)$ contains a Hamilton cycle and hence $B(X, Y)$ contains a Hamilton cycle. This in turn implies that $D$ contains an anti-directed Hamilton cycle.

\section{Proofs of Theorems 7, 8 and 9}

In [1] the following Type 1 reduction was used to prove Theorem 7. 
Type 1 reduction. Let $D$ be a directed graph and let $V(D)=P \cup Q$ be a partition of $V(D)$. Let $p$ and $p^{\prime}$ be distinct vertices in $P$ and let $q \in Q$ such that $p q \in A(D)$ and $q p^{\prime} \in A(D)$. A Type 1 reduction applied to $D$ with respect to the vertices $p, q$, and $p^{\prime}$ produces a directed graph $D_{1}$ from $D$ with $V\left(D_{1}\right)=\left(V(D)-\left\{p, q, p^{\prime}\right\}\right) \cup\left\{q_{1}\right\}$ and with $E\left(D_{1}\right)$ obtained from $A(D)$ as follows: Delete arcs $v p \in A(D)$ for each $v \in V(D)$, delete $\operatorname{arcs} p^{\prime} v \in A(D)$ for each $v \in V(D)$, delete all arcs incident on $q$, replace arc $p v \in A(D)$ by an arc $q_{1} v$ for each $v \in V(D)$, and, replace arc $v p^{\prime} \in A(D)$ by an arc $v q_{1}$ for each $v \in V(D)$. Let $P_{1}=P-\left\{p, p^{\prime}\right\}$ and $Q_{1}=(Q-\{q\}) \cup\left\{q_{1}\right\}$. Clearly, if $D_{1}$ contains a $\left(P_{1}, Q_{1}\right)$ vertex-oriented Hamilton cycle then $D$ contains a $(P, Q)$ vertex-oriented Hamilton cycle that includes the arcs $p q$ and $q p^{\prime}$.

For the sake of completeness we include the proof of Theorem 7 here.

Proof of Theorem 7. If $j=0$, then $P=\emptyset$ and $\delta^{0}(D) \geqslant \frac{n}{2}$. Theorem 2 implies that $D$ contains a directed Hamilton cycle which is a $(\emptyset, V(D))$ vertex-oriented Hamilton cycle in $D$. Now suppose that $j \geqslant 1$. Let $p$ and $p^{\prime}$ be distinct vertices in $P$. It is easy to see that there exists $q \in Q$ such that $p q \in A(D)$ and $q p^{\prime} \in A(D)$. We now apply a Type 1 reduction to $D$ with respect to the vertices $p, q$, and $p^{\prime}$ to obtain the directed graph $D_{1}$ with partition of $V\left(D_{1}\right)$ into $V\left(D_{1}\right)=P_{1} \cup Q_{1}$, where $P_{1}=P-\left\{p, p^{\prime}\right\}$ and $Q_{1}=(Q-\{q\}) \cup\left\{q_{1}\right\}$. Now, $\left|V\left(D_{1}\right)\right|=n-2,\left|P_{1}\right|=2 j-2$, and since $\delta^{0}(D) \geqslant \frac{n}{2}+j$ we have that $\delta^{0}\left(D_{1}\right) \geqslant\left(\frac{n}{2}+j\right)-2=\frac{n-2}{2}+\frac{2 j-2}{2}$. So, we can apply a Type 1 reduction to $D_{1}$ to get the directed graph $D_{2}$ with partition $V\left(D_{2}\right)$ into $V\left(D_{2}\right)=P_{2} \cup Q_{2}$, where $P_{2}$ and $Q_{2}$ are obtained from $P_{1}$ and $Q_{1}$ in a manner similar to the one by which $P_{1}$ and $Q_{1}$ were obtained from $P$ and $Q$. Iterating this procedure a total of $j$ times yields a directed graph $D_{j}$ with $P_{j}=\emptyset$ and $Q_{j}=V\left(D_{j}\right)$ with $\left|V\left(D_{j}\right)\right|=n-2 j$ and $\delta^{0}\left(D_{j}\right) \geqslant \frac{n}{2}+j-2 j=\frac{n-2 j}{2}$. Now, Theorem 2 implies that $D_{j}$ contains a directed Hamilton cycle which in turn implies that $D$ contains a $(P, Q)$ vertex-oriented Hamilton cycle.

To prove Theorems 8 and 9 we will use the following Type 2 reduction.

Type 2 reduction. Let $D$ be a directed graph and let $V(D)=P \cup Q$ be a partition of $V(D)$. Let $p$ and $p^{\prime}$ be distinct vertices in $P$ with $p p^{\prime} \in A(D)$. A Type 2 reduction applied to $D$ with respect to the vertices $p$ and $p^{\prime}$ produces a directed graph $D_{2}$ from $D$ with $V\left(D_{2}\right)=\left(V(D)-\left\{p, p^{\prime}\right\}\right) \cup\left\{q_{2}\right\}$ and with $E\left(D_{2}\right)$ obtained from $A(D)$ as follows: Delete arcs $v p \in A(D)$ for each $v \in V(D)$, delete $\operatorname{arcs} p^{\prime} v \in A(D)$ for each $v \in V(D)$, replace arc $p v \in A(D)$ by an arc $q_{2} v$ for each $v \in V(D)$, and, replace arc $v p^{\prime} \in A(D)$ by an arc $v q_{2}$ for each $v \in V(D)$. Let $P_{2}=P-\left\{p, p^{\prime}\right\}$ and $Q_{2}=Q \cup\left\{q_{2}\right\}$. Clearly, if $D_{2}$ contains a $\left(P_{2}, Q_{2}\right)$ vertex-oriented Hamilton cycle then $D$ contains a $(P, Q)$ vertex-oriented Hamilton cycle that includes the arc $p p^{\prime}$.

Proof of Theorem 8. Let $D$ be a directed graph of order $n$. Let $V(D)=P \cup Q$ be a partition of $V(D)$ with $|P|=2 j \geqslant \frac{2}{3} n$ for some integer $j \geqslant 0$. Let $D[P]$ be the directed subgraph of $D$ induced by vertices in $P$, and let $G(P)$ be the simple graph 
underlying $D[P]$. Since $\delta^{0}(D) \geqslant \frac{n}{2}+\frac{j}{2}, 2 j \geqslant \frac{2}{3} n$, and, $|Q|=n-2 j$, we have that $\delta(G(P)) \geqslant\left(\frac{n}{2}+\frac{j}{2}\right)-(n-2 j) \geqslant j$. Hence, Theorem 1 implies that $G(P)$ contains a Hamilton cycle and hence a perfect matching $M$. Let $\left(p_{i}, p_{i}^{\prime}\right), i=1,2, \ldots, j$ be the $j$ arcs in $D[P]$ corresponding to the edges in $M$. We now successively apply $j$ Type 2 reductions to $D$ with respect to the vertices $p_{i}$ and $p_{i}^{\prime}$ for $i=1,2, \ldots, j$. Let $D^{*}$ be the directed graph obtained from $D$ after these $j$ Type 2 reductions. Then, $\mid V\left(D^{*} \mid=n-j\right.$ and since $\delta^{0}(D) \geqslant \frac{n}{2}+\frac{j}{2}$, we have that $\delta^{0}\left(D^{*}\right) \geqslant\left(\frac{n}{2}+\frac{j}{2}\right)-j=\frac{n-j}{2}$. Now, Theorem 2 implies that $D^{*}$ contains a directed Hamilton cycle which in turn implies that $D$ contains a $(P, Q)$ vertex-oriented Hamilton cycle.

We will need the following Lemma [4] in the proof of Theorem 9.

Lemma 2 [4] Let $G$ be a graph of order $n$ and let $\beta(G)$ be the maximum cardinality of a matching in $G$. Then $\beta(G) \geqslant \min \left\{\delta(G),\left\lfloor\frac{n}{2}\right\rfloor\right\}$.

Proof of Theorem 9. Let $D$ be a directed graph of order $n$. Let $V(D)=P \cup Q$ be a partition of $V(D)$ with $|P|=2 j$ for some integer $j \geqslant 0$ and with $\frac{1}{3} n \leqslant 2 j \leqslant \frac{2}{3} n$. Let $2 j=\frac{1}{3} n+k, 0 \leqslant k \leqslant \frac{1}{3} n$. Let $D[P]$ be the directed subgraph of $D$ induced by vertices in $P$, and let $G(P)$ be the simple graph underlying $D[P]$. Since $\delta^{0}(D) \geqslant \frac{2}{3} n$ and $|Q|=n-2 j$, we have that $\delta(G(P)) \geqslant \frac{2}{3} n-(n-2 j)=2 j-\frac{1}{3} n=k$. Since $2 j \leqslant \frac{2}{3} n$, we have that $k=2 j-\frac{1}{3} n \leqslant j=\frac{|V(G(P))|}{2}$. Lemma 2 implies that $G(P)$ contains a matching $M$ with $|M|=\lceil k\rceil$. Let $\left(p_{i}, p_{i}^{\prime}\right), i=1,2, \ldots,\lceil k\rceil$ be the $\lceil k\rceil$ arcs in $D[P]$ corresponding to the edges in $M$. We now successively apply $\lceil k\rceil$ Type 2 reductions to $D$ with respect to the vertices $p_{i}$ and $p_{i}^{\prime}$ for $i=1,2, \ldots,\lceil k\rceil$. Let $D^{*}$ be the directed graph obtained from $D$ after these $\lceil k\rceil$ Type 2 reductions. Then, $\mid V\left(D^{*} \mid=n-\lceil k\rceil\right.$ and since $\delta^{0}(D) \geqslant \frac{2}{3} n$, we have that $\delta\left(D^{*}\right) \geqslant \frac{2}{3} n-\lceil k\rceil$. Let $P^{*}=P-\cup_{i=1}^{\lceil k\rceil}\left\{p_{i}\right\}-\cup_{i=1}^{\lceil k\rceil}\left\{p_{i}^{\prime}\right\}$ and let $Q^{*}=V\left(D^{*}\right)-P^{*}$. We have that $\left|P^{*}\right|=2 j-2\lceil k\rceil=\frac{1}{3} n+k-2\lceil k\rceil$. Hence, $\delta\left(D^{*}\right) \geqslant \frac{2}{3} n-\lceil k\rceil \geqslant \frac{1}{2}\left|V\left(D^{*}\right)\right|+\frac{1}{2}\left|P^{*}\right|$. Now, Theorem 7 implies that $D^{*}$ contains a $\left(P^{*}, Q^{*}\right)$ vertex-oriented Hamilton cycle which in turn implies that $D$ contains a $(P, Q)$ vertex-oriented Hamilton cycle.

\section{Conclusion}

We summarize the results given in this paper as follows. Let $D$ be a directed graph of order $n$ and let $V(D)=P \cup Q$ be a partition of $V(D)$ with $|P|=p$, and $p$ being even. By Theorems 7,8 , and 9 , with $f(n, p)$ as defined below, if $\delta^{0}(D) \geqslant f(n, p)$ then $D$ contains a $(P, Q)$ vertex-oriented Hamilton cycle.

$$
f(n, p)= \begin{cases}\frac{1}{2} n+\frac{1}{2} p, & \text { if } 0 \leqslant p \leqslant \frac{1}{3} n \\ \frac{2}{3} n, & \text { if } \frac{1}{3} n \leqslant p \leqslant \frac{2}{3} n \\ \frac{1}{2} n+\frac{1}{4} p, & \text { if } \frac{2}{3} n \leqslant p \leqslant n .\end{cases}
$$

In the case when $p=n$, we can do better than the previous statement promises. Theorem 6 gives us that $f(n, p)=\frac{2}{3} n$ if $p=n$, thus, it is natural to expect that the lower bounds 
on $\delta^{0}(D)$ that guarantee a $(P, Q)$ vertex-oriented Hamilton cycle can be significantly improved when $p$ is relatively large.

\section{References}

[1] K. N. Badheka, M. J. Plantholt, and S. K. Tipnis, On a well-spread halving of directed multigraphs, Ars Combinatoria 83 (2007) 257-265.

[2] J.A. Bondy and U.S.R. Murty, Graph Theory, Springer, GTM 244 (2008).

[3] Dirac G.A., Some theorems on abstract graphs, Proc. London Math. Soc. 2 (1952) 69-81.

[4] P. Erdös and L. Pósa, Publ. Math. Debrecen 9 (1962) 3-12.

[5] F. Harary, Graph Theory, Addison-Wesley, Reading, MA(1969).

[6] A. Ghouila-Houri, Une condition suffisante d'existence d'un circuit Hamiltonien, C.R. Acad. Sci. Paris 156 (1960) 495-497.

[7] D.D. Grant, Anti-directed Hamilton cycles in digraphs, Ars Combinatoria 10 (1980) 205-209.

[8] R. Häggkvist and A. Thomason, Oriented Hamilton cycles in digraphs, J. of Graph Theory, 19, No. 4, (1995) 471-479.

[9] R. Häggkvist and A. Thomason, Oriented hamilton cycles in oriented graphs, Combinatorics, geometry and probability Cambridge University Press, Cambridge 1997, MR 1476456.

[10] Mao-cheng Cai, A counterexample to a conjecture of Grant, Discrete Mathematics 44 (1983) 111.

[11] J. Moon and L. Moser, On Hamiltonian bipartite graphs, Israel J. Math. 1 (1963) 163-165. 